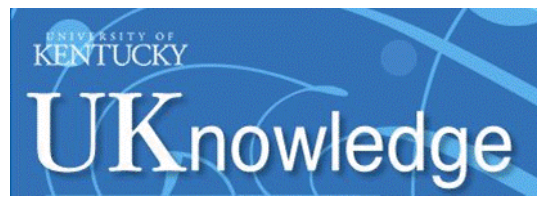

University of Kentucky

UKnowledge

Chemical and Materials Engineering Faculty

Publications

Chemical and Materials Engineering

8-2014

\title{
Surface Structure of Catalytically-active Ceria Nanoparticles
}

\author{
Xing Huang \\ University of Kentucky, jerry.x.huang@uky.edu \\ Matthew J. Beck \\ University of Kentucky, mjbe223@uky.edu
}

Follow this and additional works at: https://uknowledge.uky.edu/cme_facpub

Right click to open a feedback form in a new tab to let us know how this document benefits you.

\section{Repository Citation}

Huang, Xing and Beck, Matthew J., "Surface Structure of Catalytically-active Ceria Nanoparticles" (2014). Chemical and Materials Engineering Faculty Publications. 3.

https://uknowledge.uky.edu/cme_facpub/3

This Article is brought to you for free and open access by the Chemical and Materials Engineering at UKnowledge. It has been accepted for inclusion in Chemical and Materials Engineering Faculty Publications by an authorized administrator of UKnowledge. For more information, please contact UKnowledge@lsv.uky.edu. 


\section{Surface Structure of Catalytically-active Ceria Nanoparticles}

\section{Digital Object Identifier (DOI)}

http://dx.doi.org/10.1016/j.commatsci.2014.04.037

\section{Notes/Citation Information}

Published in Computational Materials Science, v. 91, p. 122-133.

Per the publisher Elsevier: "NOTICE: this is the author's version of a work that was accepted for publication in Computational Materials Science. Changes resulting from the publishing process, such as peer review, editing, corrections, structural formatting, and other quality control mechanisms may not be reflected in this document. Changes may have been made to this work since it was submitted for publication. A definitive version was subsequently published in Computational Materials Science, v. 91, (2014). DOI: 10.1016/j.commatsci.2014.04.037" 


\title{
Surface structure of catalytically-active ceria nanoparticles
}

\author{
Xing. Huang ${ }^{\mathrm{a}}$, Matthew J. Beck ${ }^{\mathrm{a}, * *}$ \\ ${ }^{a}$ Department of Chemical \& Materials Engineering, University of Kentucky, Lexington, \\ KY 40506
}

\begin{abstract}
Catalytic mechanisms, and therefore activity, depend on the structure of catalyst surfaces. In turn, surfaces may reconstruct and/or exhibit local configurations that vary from bulk composition and structure. $\mathrm{CeO}_{2}$ (ceria) is a redox catalyst of interest in numerous automotive, energy and, increasingly, biomedical applications. Previous studies aimed at understanding catalytic mechanisms on ceria have limited consideration to systems with bulk-like stoichiometric or sub-stoichiometric surfaces. Here we summarize previous computational studies on ceria surfaces, nanoclusters, and nanoparticles, and highlight challenges in constructing physically-representative ceria nanoparticle (CNP) structures. Setting aside assumptions of bulk-like stoichiometric or sub-stoichiometric ceria surface terminations, we report results of DFT $+U$ calculations and show that sufficiently small CNPs are not bulk-terminated, but rather are stabilized by the formation of $\mathrm{O}_{x}^{q}$ groups $(-2 \leq \mathrm{q} \leq 0, \mathrm{x} \leq 3)$ at corners, edges, and $\{100\}$ facets. These surface structures, not the annihilation and regeneration of O-vacancies, may directly control reduction/oxidation catalysis at CNPs below a critical size. As anion groups other than $\mathrm{O}_{x}^{q}$ groups could be incorporated in stable CNP
\end{abstract}

\footnotetext{
${ }^{*}$ Corresponding author. Address: 157 F. Paul Anderson Tower, Lexington, KY, USA, 40506. Tel.: +1 8592570039.

**E-mail address: mjbe223@uky.edu (M. J. Beck)
} 
surfaces, this suggests the possibility of tailoring small CNP structures and mechanisms for particular catalytic reactions.

Keywords: Ceria nanoparticles, Density functional theory, Surface

structure

\section{Introduction}

Catalysts are essential engineering materials used to promote specific chemical reactions in a host of applications and processes. Typical catalysts influence target reactions by enhancing the binding or localization of reactant molecules at catalyst surfaces while lowering reaction barriers and allowing desorption of product molecules. Ideal catalysts have high activity, specificity and stability, as well as low contamination rates under operating conditions. Various pure metals and, increasingly, metal oxides are widely applied as catalysts. Reversible reduction/oxidation catalysts have particular technological value in energy, environmental and biomedical applications. Cerium dioxide $\left(\mathrm{CeO}_{2}\right.$, ceria) has served as an excellent reduction/oxidation catalyst for 30 years. Ceria has been shown to have wide applications in environmental remediation, energy generation and biomedicine, in addition to other important applications in microelectronics, optical films, gas sensors, and polishing materials [? ? ? ? ? ? ? ? ? ? ? ? ].

The effects of a catalyst on a chemical reaction are necessarily surface effects. That is, the physical and chemical action of a catalyst occurs at the interface between the catalyst and its environment-namely, at the catalyst's surfaces. Enhancing or tailoring catalytic behavior, therefore, first requires an understanding of the structure and properties of the catalyst's surfaces. In ceria or ceria-supported catalysis the discovery that bulk cubic fluorite ceria can be reduced through the formation of O-vacancies and 
reversibly (re-)oxidized via the annihilation of O-vacancies has led to the development of a general understanding that the presence of (or potential to form) O-vacancies on ideal, bulk-terminated ceria surfaces drives the catalytic activity of ceria in reduction/oxidation reactions. In fact, explanations for the mechanism of ceria catalysis have, to date, assumed ideal, bulk-like ceria surface structures, or minor perturbations thereof. But does this picture apply to ceria nanoparticle (CNP) systems? Do the catalytically active surfaces of CNPs actually exhibit ideal, bulk-like surface terminations?

In this paper we report DFT $+U$ calculation results demonstrating that sufficiently small CNPs are not bulk-terminated, but instead exhibit stable surface configurations characterized by the formation of chemically bound $\mathrm{O}_{x}^{q}$ groups. In presenting these results we summarize previous computational efforts to discern the detailed structures and properties of experimentallyrelevant ceria facets and CNPs. We highlight how previous studies have generally limited consideration to structures with bulk-like surface terminations and primarily focus on the potential role of lattice O-vacancies as active surface sites for ceria catalysis. We discuss the inherent challenges in constructing physically-representative structures for computing CNP surface properties, and note that the complexity underlying these challenges motivates an exploration of CNP structures that is not limited by assumptions of bulk-like CNP surface terminations. We then give a detailed description of our computational methods, including a discussion of how initial CNP structures were generated and the computational tools and techniques used to relax these structures to their ground state (lowest energy) configurations. We derive a robust expression for the excess energy of formation capable of characterizing - and allowing comparisons between - the stability of surface configurations on CNPs of various shapes and sizes. 
The present DFT $+U$ results show that sufficiently small CNPs are stabilized by the formation of bound $\mathrm{O}$ pairs and triples at corners, edges and $\{100\}$ facets in both O-rich and O-lean environments. We further show that a straightforward model accounting for formal charges and the nonstoichiometry of CNP corners, edges and $\{100\}$ facets predicts a transition from larger, net reduced $\left(\mathrm{CeO}_{2-x}\right)$ particles which would exhibit bulklike surface terminations (as assumed by the present understanding of ceria catalysts), to smaller, oxidized $\left(\mathrm{CeO}_{2+x}\right)$ particles with $\mathrm{O}_{x}^{q}$-based surface terminations (shown to be stable here). The stability of $\mathrm{O}_{x}^{q}$-terminated CNPs demonstrates that, below a critical size, the catalytic activity and O-storage capacity of CNPs is controlled not by the thermodynamics and kinetics of O-vacancies, but rather directly by surface structures that incorporate molecular anions. In turn, the detailed structures of catalyticallyactive CNP surfaces - specifically including the type and distribution of surface anion groups - are controlled by surface chemistry, and therefore by experimentally-controllable environmental conditions like solvent composition, $\mathrm{pH}$, and CNP surface functionalization.

\section{Background}

\subsection{Ceria catalysis}

The excellent catalytic properties of ceria were first harnessed in the late 1970s when ceria was utilized for the treatment automobile exhaust, serving as a key component in three-way catalysts (TWC) [? ? ]. During the same time period, studies on the phase diagram of the cerium oxide system were carried out. It was discovered that bulk ceria (cubic fluorite $\mathrm{CeO}_{2}$ ) can be reduced at elevated temperatures to form a range of O-deficient, 
non-stoichiometric oxides $\left(\mathrm{CeO}_{2-x}\right)$, referred to collectively as "reduced" ceria. In addition, it was shown that ceria is able to reversibly transform among these various reduced phases [? ? ? ]. Further studies [? ? ? ] directly exploring these transformations found that bulk ceria retains a cubic fluorite structure despite removal of $25 \%$ of lattice $\mathrm{O}$ atoms when subjected to reduction below $900 \mathrm{~K}$. While these studies noted an anomalous lattice expansion during reduction, they found that O-vacancy-containing reduced ceria could be completely recovered to stoichiometric $\mathrm{CeO}_{2}$ when exposed to an oxidizing environment, even at room temperature.

These discoveries implied that ceria can store $\mathrm{O}$ by adsorbing it (via the annihilation of O-vacancies) under O-rich conditions, and controllably release it by (re-)forming O-vacancies under O-poor or reducing conditions. The number of $\mathrm{O}^{\mathrm{II}}$ anions (per volume or unit mass) that can be reversibly extracted and recovered during a particular reaction (e.g., oxidation or reduction of $\left.\mathrm{CO} / \mathrm{CO}_{2}\right)$ is quantitatively characterized as the "oxygen storage capacity" (OSC) of a ceria system [? ? ]. Hence, ceria has two related, technologically important capabilities: the ability to strongly catalyze redox reactions, and the ability to serve as a solid state oxygen buffer for redox reactions.

Since the 1970s substantial experimental and computational effort has been expended to understand, control, and ultimately optimize ceria's natural ability to both support [? ? ] and directly catalyze [? ? ? ? ? ? ] redox reactions. Over the course of these investigations the experimentally measurable OSC of ceria and ceria-containing systems has become a kind of litmus test for catalytic activity. That is, the realizable potential of a ceria system to form and annihilate $\mathrm{O}$-vacancies during reduction and oxidation has become both the lens through which the general catalytic properties of 
all ceria-based materials are viewed, and the default basis for understanding all reduction/oxidation mechanisms in such materials.

For bulk ceria systems, the existence of O-vacancy-containing cubic fluorite "reduced ceria" phases and low-barrier reversible transformation paths between them (simply the formation/annihilation of O-vacancies) suggests a straightforward, "four-step" mechanistic hypothesis explaining ceria's excellent catalytic activity: (1) formation of bulk O-vacancies and associated Ce $\mathrm{III}^{\mathrm{II}}$ cations, (2) migration of bulk $\mathrm{Ce}^{\mathrm{III}}$ cations to a ceria surface, (3) adsorption of O-molecules at surface $\mathrm{Ce}$ III cations, and (4) interaction of reactant molecules with adsorbed O-molecules leading to catalyzed product formation and (re-)oxidation of (surface) $\mathrm{Ce}^{\mathrm{III}}$ cations.

The above "four-step" mechanism assumes two key points: (i) that reactive $\mathrm{Ce}^{\mathrm{III}}$ cations are present only in conjunction with vacant $\mathrm{O}^{\mathrm{II}}$ lattice sites, and (ii) that the intrinsic ceria surface itself has no direct role in catalytic reactions - that is, redox catalysis by ceria requires that a vacant $O$ lattice site must migrate, along with its concomitant $\mathrm{Ce}$ III cations, to the surface, and that this (surface) defect structure, not any intrinsic feature of the stable surface itself, is catalytically active. These assumptions have important consequences, namely implying that optimizing the activity of ceria catalysts requires increasing the concentration of surface O-vacancies (e.g., by reducing surface O-vacancy formation energies, or increasing bulk O-vacancy concentrations). Given that these implications have driven much of the recent research on optimizing or enhancing ceria and ceria-supported catalysts, it is worth considering under what conditions the assumptions leading to the "four-step" mechanism are applicable.

The first assumption (that $\mathrm{Ce}$ III cations are present in conjunction with O-vacancies) is well-justified in bulk ceria systems with fixed charge, where 
reducing bulk ceria (that is, generating $\mathrm{Ce}^{\mathrm{III}}$ cations) while maintaining charge neutrality requires the formation of one lattice O-vacancy for every two $\mathrm{Ce}^{\mathrm{III}}$ cations. On this basis, it is a general practice to consider the reduction of ceria, the creation of $\mathrm{Ce}^{\mathrm{III}}$ cations, and/or the creation of lattice O-vacancies to be equivalent processes in bulk ceria. The existence of large numbers of reactive $\mathrm{Ce}$ III cations in ceria systems is itself justified by the existence of a range of sub-stoichiometric cubic fluorite reduced ceria phases $\left(\mathrm{CeO}_{2-x}\right)$ which can be reversibly oxidized or reduced [? ? ? ? ].

The second assumption upon which the "four-step" mechanism is based, that catalytically-active surface sites are $\mathrm{Ce}^{\mathrm{III}}$ cations present as lattice O-vacancies on ceria surfaces, implies by extension that stable ceria surface structures do not intrinsically exhibit $\mathrm{Ce}^{\mathrm{III}}$ cations, and that no other stable surface structures (other than surface $\mathrm{Ce}^{\mathrm{III}}$ cations) are catalytically active. An argument can be made that this assumption is applicable for ideal, bulkterminated surfaces on semi-infinite ceria samples, particularly on the basis of STM images of such surfaces revealing ideal, unreconstructed structures populated with finite concentrations of vacant lattice $\mathrm{O}$ sites [? ]. The "four-step" mechanism of ceria catalysis (and the assumptions it is based upon) is therefore well-supported for bulk samples (or more correctly, for structures with surfaces well-approximated as ideal, unreconstructed, semiinfinite and stoichiometric bulk cuts). But the direct translation of the above assumptions to nanoparticle structures ignores the possibility of completely new atomic arrangements and catalytic mechanisms accessible only on the surfaces of nanoscale systems. 


\subsection{Previous computational results}

\subsubsection{Semi-infinite ceria surfaces}

Given the critical role of O-vacancies enabling the catalysis by ceria, various computational studies aimed at understanding O-vacancy formation in bulk ceria and at ideal bulk ceria surfaces have been carried out. In 1992, Sayle et al. [? ? ] investigated the structures and energetics of O-vacancy formation in stoichiometric bulk ceria, and on ideal, stoichiometric $\{111\}$, $\{110\}$ and $\{310\}$ ceria surfaces. Sayle found that O-vacancy formation was energetically more favorable on ceria surfaces than in bulk, and more favorable on $\{110\}$ and $\{310\}$ surfaces than on $\{111\}$ surfaces. Later, in addition to confirming the 1992 Sayle results, Conesa [? ] studied the structure and energetics of three other low-indexed ceria surfaces: stoichometric $\{211\}$ surface as well as non-stoichiometric $\{100\}$ and $\{210\}$ surfaces. This work found that $\{211\}$ surface relaxed into stepped $\{111\}$ configuration. Besides individual O-vacancies, O-vacancy pairs were found to be formed on lowindexed ceria surfaces, and they were more stable on $\{111\}$ surface than $\{110\}$ surface or $\{100\}$ surface.

The results of Sayle et al. and Conesa were generated using empirical interatomic potentials which required that the oxidation state of Ce cations be assumed and fixed. That is, such methods could not be used to calculate the low-energy distribution of electrons - as required to predict the distribution of Ce oxidation states for a given atomic configuration. In 2002, Skorodumova used DFT calculations to lift this limitation and directly explore the electronic structure of O-vacancies in ceria [? ]. Considering both ceria and a cubic fluorite reduced ceria structure with $\mathrm{Ce}_{2} \mathrm{O}_{3}$ stoichiometry, Skorodumova concluded that O-vacancies prefer to be located in the crystal 
bulk - in contrast to previous semi-empirical results [? ]. Importantly, they also concluded that the two excess electrons associated with a lattice $\mathrm{O}$ atom removed to form a vacancy localize into the $4 \mathrm{f}$ states of two of the four Ce atoms nearest neighbor to the vacant $\mathrm{O}$ lattice site. Therefore, the redox properties of ceria were found to depend upon electron transitions from delocalized $O 2 p$ states to localized $C e \& f$ states that occur during O-vacancy formation. In addition, these results imply that identifying and characterizing $\mathrm{O}$-vacancies in ceria is equivalent to identifying and characterizing $\mathrm{Ce}^{\mathrm{III}}$ cations. That is, because $\mathrm{Ce}$ III cations are preset in stoichiometric ceria only if O-vacancies are present, and, when present, are physically adjacent to an O-vacancy, the thermodynamics and kinetics of O-vacancies are the thermodynamics and kinetics of $\mathrm{Ce}$ III cations.

\subsubsection{Small ceria clusters and ceria nanoparticles (CNPs)}

To explore whether nanoceria (broadly meaning ceria materials with one or more dimension on the nanoscale) behaves similarly to larger ceria samples, computational studies have considered O-vacancy properties in small ceria clusters and nanoparticles. In 1996 Cordatos et al.[? ], again employing interatomic potentials, found that only large clusters formed fluorite crystallites with well-defined facets. Smaller clusters tended to form spherical agglomerations, while nanocrystals formed with $\{111\}$ facets. Cluster stability increased nonlinearly with cluster size. The reducibility of ceria clusters was assessed based on the difference in lattice energy between $\mathrm{Ce}_{n} \mathrm{O}_{2 n}$ and $\mathrm{Ce}_{n} \mathrm{O}_{2 n-1}$ clusters, that is, by calculating an effective O-vacancy formation energy. Reducibility (O-vacancy formation energy) was found to be structure sensitive, but generally decreased (increased) with increasing cluster size. In 1998, Vyas et al. [? ] predicted that stoichiometric ceria crystallites 
exhibited an octahedral morphology with $\{111\}$ facets, in agreement with the Cordatos' previous findings.

Using similar interatomic potentials, Sayle et al. [? ] conducted molecular dynamics simulations of ceria nanoparticles as large as $10 \mathrm{~nm}$. Despite beginning with completely different initial shapes, all three CNPs considered by Sayle et al., relaxed to similar shapes: truncated octahedrons comprised of eight $\{111\}$ facets and $\{100\}$ mini-facets at the six corners. It is notable that the $\{110\}$ surfaces previously predicted [? ? ? ] in semi-infinite slab calculations as more stable than $\{100\}$ surfaces but less stable than $\{111\}$ surfaces disappeared in small CNPs, leaving behind only $\{111\}$ and $\{100\}$ surfaces. The resulting $\{100\}$-truncated octahedral CNPs agreed well with experimentally determined shapes of certain synthesized CNPs [? ].

As noted above, the use of empirical interatomic potentials requires the oxidation state of Ce cations to be fixed a priori. Therefore, calculated lattice energies of sub-stoichiometric (reduced) nanoparticles and calculated reducibilities are constrained by an external assignment of $\mathrm{Ce}^{\mathrm{III}}$ cation locations. In reduced clusters, Cordatos placed $\mathrm{Ce}^{\mathrm{III}}$ cations at the nanoparticle surfaces in generally unpaired arrangements, in contrast to Skorodumova's findings [? ] (reported six years after Cordatos' study, see above). In 2006, density functional theory (DFT) based calculations not requiring a priori specification of the oxidation state of Ce cations were applied to study ceria clusters. Tsunekawa et al. [? ], using GGA calculations, calculated the electronic density of states (eDOS) and band structure of a fully oxidized $\left(\mathrm{CeO}_{2}\right)_{13}$ cluster and a fully reduced $\left(\mathrm{CeO}_{1.5}\right)_{13}$ cluster. Subsequently, both $\mathrm{LDA}+U$ and GGA $+U$ calculations [? ? ? ] were employed to investigate the geometric and electronic structures of small ceria clusters and their reducibility. They found the O-vacancy formation energy to be much lower 
in ceria clusters than in the bulk ceria, implying higher concentrations of $\mathrm{Ce}^{\mathrm{III}}$ cations, and therefore, according to the "four-step" mechanism, better catalytic properties. The chemical reactivity of differently configured Ce and $\mathrm{O}$ atoms was also examined, and variations in chemical reactivity were found to be associated with dissimilar coordination states of various atoms $[?]$.

In recent years, various authors have reported DFT-based studies of ceria nanocrystals with distinct surface facets[? ? ? ? ? ? ? ]. These studies have been limited to $\{111\}$-terminated octahedral CNPs which are partially reduced $\left(\mathrm{CeO}_{2-x}\right)$ due to the sub-stoichiometric (meaning O-deficient) nanoparticle edges and corners, as well as both reduced and stoichiometric cuboctahedral CNPs with truncated corners consisting of small O-terminated $\{100\}$ facets. Of specific interest in these studies has been the formation energy of O-vacancies as a function of $\mathrm{CNP}$ sizes and $\mathrm{Ce}^{\mathrm{III}}$ versus $\mathrm{Ce}^{\mathrm{IV}}$ cation ratios [? ? ? ? ]. The major conclusion drawn from these efforts are that (1) it is easier to remove low-coordinated $\mathrm{O}$ atoms to form Ovacancies, (2) $\mathrm{Ce}^{\mathrm{III}}$ cations (present due to non-stoichiometric CNP edges, not as bulk O-vacancies) are located at the edges and corners of CNPs, whereas $\mathrm{Ce}^{\mathrm{IV}}$ cations are located at fully O-coordinated (bulk-like) sites, and (3) the formation energy of O-vacancies decreased with increasing CNP size and decreasing $\mathrm{Ce}^{\mathrm{III}}$ versus $\mathrm{Ce}^{\mathrm{IV}}$ cation ratio. Taken together, this implies that for a CNP of given size, there exists an equilibrium concentration of O-vacancies at finite temperature, and that CNPs of a certain size are expected to be most favorable for O-vacancy formation.

These studies have also sought to identify a "size-converged" model CNP, that would be as small as possible to minimize computational cost, but large enough to be representative of experimentally observed properties [? ]. Us- 


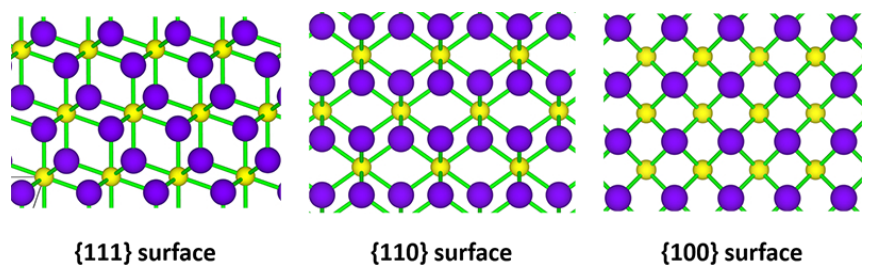

Figure 1: Atomic structure of of $\{111\},\{110\}$ and $\{100\}$ ceria surfaces. Purple balls represent $\mathrm{O}$ atoms and yellow balls represent Ce atoms.

ing the adsorption energy of CO molecules on a CNP as a test observable property, Migani et al. find that CNP properties become essentially invariant (variations within $\approx 0.1 \mathrm{eV}$ ) for CNPs with diameters of $1 \mathrm{~nm}$ and greater. It is worth noting that only one study considers the energetics of oxidized CNPs, and in that case considers only the addition of a small number of single, isolated $\mathrm{O}$ atoms at cubic fluorite surface lattice sites [? ]. The authors conclude for the limited structures and compositions considered that oxidized CNPs are less stable than partially reduced and stoichiometric CNPs. They note that the oxidized nanoparticles $\left(\mathrm{Ce}_{13} \mathrm{O}_{32}\right.$ and $\left.\mathrm{Ce}_{38} \mathrm{O}_{80}\right)$, containing 6 and 4 excess $\mathrm{O}$ atoms respectively, exhibited isolated $\mathrm{O}^{-1}$ anions ("peroxide-like $\mathrm{O}$ atoms"), whose instability is likely reflected in the relative increase in the CNP formation energy.

\subsubsection{Summary}

Previous atomistic calculations have examined both semi-infinite ceria surfaces and various ceria nanoclusters and nanocrystallites. These studies have been limited to structures with bulk-like surface configurations (see Fig. 1). Calculations on stoichiometric semi-infinite ceria surfaces (e.g., $\{111\},\{110\}$, and $\{310\})$ have, in fact, assumed ideal bulk terminations. Calculations on non-stoichiometric ceria surfaces (e.g., $\{100\}$ and $\{211\}$ ) 
were first conducted by enforcing net stoichiometry in the calculation cell by constructing asymmetric slabs with differing top and bottom surfaces (namely one O-terminated surface and an opposite Ce-terminated surface) [? ? ]. The resulting asymmetric computational cells result in electronic dipoles perpendicular to the slab surfaces. These dipoles distort the calculated electronic structure and give rise to errors in calculated surface and O-vacancy formation energies. This issue has been addressed by assuming symmetric slabs with model (top and bottom) surface structures composed of arbitrarily assigned arrangements of vacant and filled surface $\mathrm{O}$ lattice sites chosen to satisfy net stoichiometry (e.g., choosing half the surface $\mathrm{O}$ lattice sites to be unoccupied). While this both avoids the issue of $\mathrm{Ce}^{\mathrm{III}}$ cations present natively in systems with non-stoichiometric surfaces (in contrast to their presence in reduced ceria as a result of O-vacancy formation) and eliminates the problem of surface dipoles due to asymmetric slabs, these structural models do not address the issue of possible surface reconstructions, including those that may involve non-stiochiometric surface terminations.

Atomistic calculations on ceria nanocrystals have focused on stoichiometric or near-stoichiometric (particularly partially-reduced) octahedral, cuboctahedral and tetrahedral CNPs, again, with bulk-like surface terminations. Calculated results show that $\mathrm{Ce}^{\mathrm{III}}$ cations, when present, are generally under-coordinated Ce atoms (e.g., those neighboring discrete O-vacancies, or those at under-coordinated surface, edge or corner sites) and that formation of O-vacancies involves electron transfers from $O 2 p$ states to $C e$ ff states. Beyond this, calculations on faceted CNPs show that the O-vacancy formation energy, while generally lower at CNP surfaces than in bulk, is highly dependent on the CNP shape and composition, and that surface composi- 
tion itself directly influences the number and distribution of $\mathrm{Ce}$ III cations. While individual results suggest various low-energy overall configurations (combinations of CNP size, shape and composition), no definitive consensus has emerged to define a computational predicted configuration for actual CNP catalysts. Critically, CNP calculations to date have not systematically explored the possibility of non-bulk-like CNP surface terminations, and have frequently limited consideration of possible CNP configurations on the basis of bulk stoichiometry.

\subsection{Challenges in computational modeling of ultra-small CNPs}

Computing properties that are quantitatively comparable to experimental results requires the construction of realistic, physically-representative structures as calculation inputs. For bulk crystalline systems this is relatively straightforward as well-established experimental techniques have been used to determine precise unit cell configurations for most common materials (including, of course, the two bulk structures of ceria [? ]). Systems containing surfaces introduce the added complexity of determining (1) the local (surface) concentration of atoms and (2) their local configuration at the surface itself. For crystal facets, these complexities are somewhat mitigated by the availability of experimental techniques (e.g. LEEM) capable of determining surface symmetry [? ? ]. Also, the contiguous nature of bulk crystal facets generally constrains configurational complexity to identifiable rearrangements of the underlying bulk structure. Even so, surface structure (and composition) determination is an active and challenging research area [?

For nanoparticles the complexities of bulk surface systems are only exacerbated by the necessity of selecting the correct set of surfaces that define 
the three-dimensionally constrained structure. This, of course, translates as needing to know the actual shape of a nanoparticle. As an extension, not only must the local arrangements of atoms in surface facets be determined, but also the local arrangement and distribution of atoms at facet intersections (edges and corners), and the changes in atomic configurations on surface facets due to nearby edges. Finally, for small nanoparticles, large surface-to-volume ratios can even cause changes in the internal bulk atomic arrangement within the nanoparticle.

In CNPs, nanoparticle shape has been well-characterized experimentally, with TEM and XRD studies reporting octahedral, cuboctahedral and cubiodal shapes [? primarily report that such CNPs have $\{100\}$ and/or $\{111\}$ facets [? ? ? ? ? ? ? ]. In combination with calculated results for semi-infinite slabs (which find that $\{111\},\{110\}$ and $\{100\}$ surfaces have the lowest energy [? ? ? ? ]), this existing body of experimental work has motivated quantum mechanical calculations of CNPs to focus on octahedral and cuboctahedral CNPs similar to those observed experimentally [? ? ? ? ]. The availability of experimental results to inform atomistic calculations with regards to nanoparticle shape is in stark contrast to the lack of experimental results addressing the question of the detailed atomic arrangements and relative compositions of atoms at CNP surfaces.

The lack of experimental information regarding detailed arrangements of atoms at CNP surfaces leads immediately to a key challenge in constructing input atomic configurations for the computational modeling of CNPs. Consider building a $\sim 1 \mathrm{~nm}\{111\}$-terminated octahedral CNP, similar to those observed experimentally [? ? ? ? ]. Beginning with a bulk cubic fluorite ceria structure, we may cut eight intersecting $\{111\}$ planes to form an octa- 
hedron with a diameter of $\sim 1 \mathrm{~nm}$. The structure has 44 Ce atoms, and the atomic arrangement internal to the CNP is stoichiometric (as these atoms are simply arranged in the ideal bulk cubic fluorite structure). In addition, the $\{111\}$ surfaces themselves have a stoichiometric O-termination (as observed via, e.g., STM [? ]). But, as shown in Figure 2f, the edges and corners of the CNP are not stoichiometric, and require that a configurational choice be made. If all edge and corner $\mathrm{O}$ sites are occupied (as shown in Figure 2f, where blue balls are $\mathrm{O}$ atoms at facet, edge, and corner $\mathrm{O}$ lattice sites), the structure contains eighty excess $\mathrm{O}$ atoms $\left(\mathrm{Ce}_{44} \mathrm{O}_{160}\right)$. How do we best model real \{111\}-terminated CNPs? Previous studies focusing on ideal, bulk-terminated CNP surfaces and stoichiometric CNPs have removed all surface $\mathrm{O}$ atoms (blue balls in Figure 2f) and four (of six) arbitrarily chosen corner Ce atoms to force a stoichiometric $\mathrm{Ce}_{40} \mathrm{O}_{80}$ structure [? ? ]. While a single result finds that CNPs with isolated excess $\mathrm{O}$ atoms at bulk $\mathrm{O}$ lattice sites are energetically unfavorable [? ] and a number of calculations have considered partially reduced CNPs [? ? ? ? ? ? ? ], no comprehensive exploration of the stability of CNPs versus $\mathrm{O}$ facet/edge/corner concentration has been completed.

Beyond this (relatively) straightforward case of octahedral $\{111\}$-terminated CNPs, we must also consider other CNP shapes that have been experimentally observed, particularly those reported to have high catalytic activity. CNP cubes, for example, are bounded by six $\{100\}$ facets, have been observed experimentally, and $\{100\}$ surfaces are reported to have particularly high activity [? ? ? ? ? ]. In this case the $\{100\}$ surface itself does not have a stoichiometric termination, and even greater configurational gymnastics must be contemplated if we are to determine the stable CNP configuration. For example, a $\sim 1 \mathrm{~nm}$ CNP cube with all surface O lattice sites occupied 
has composition $\mathrm{Ce}_{32} \mathrm{O}_{121}$. To construct a stoichiometric CNP requires the removal of $57 \mathrm{O}$ atoms. As there are an even number of facets, edges and corners on a cubic CNP, there is no possible way to achieve stoichiometry by removing $\mathrm{O}$ atoms evenly from facets, edges and/or corners!

Ultimately, as catalysis is a surface process, understanding the atomistic concentrations and configurations at CNP surfaces is critical to accurately modeling their catalytic mechanisms. In general, it is not possible to intuit the stable concentration or configuration of surface species, as highlighted by the challenges of constructing atomistic input structures. This is even more an issue when it is noted that catalysts are both synthesized and applied in open systems where the environment the nanoparticle catalyst is in can serve as a source and/or sink of atoms or molecules. Therefore, in the absence of clear experimental information as to the detailed surface structures of real CNPs, computational attempts to characterize CNP properties must systematically investigate CNP structures without assuming particular concentrations or configurations of atoms at CNP surfaces. In the following sections we directly address this challenge. While constraining the present work to experimentally observed CNP shapes and considering only CNPs in pure $\mathrm{O}$ environments, we systematically examine the structure and energy of $\sim 1-1.5 \mathrm{~nm}$ CNPs with unconstrained surface concentrations and configurations of $\mathrm{O}$ atoms.

\section{Computational details}

\subsection{Calculation methodology and settings}

In the following we apply DFT $+U$ calculations to explore the properties of faceted, nanometer-scale CNPs with various shapes similar to those 
observed experimentally. To accurately characterize relaxed atomic geometries, valence electron charge densities, and total energies of CNPs with various compositions and surface configurations, we apply DFT $+U$ calculations as implemented in the VASP code [? ]. The accuracy and efficacy of this method as applied to the structure and energetics of CNPs has been previously demonstrated as discussed above.

For the present calculations, exchange and correlation were described both at the level of local density approximation (LDA) using the CeperleyAlder functional [? ] as parametrized by Perdew and Zunger [? ], and at the level of the generalized gradient approximation (GGA) using the PerdewBurke-Ernzerhof (PBE) formalism [? ]. The Ce 4 f5s5p5d6s as well as $O$ $2 p 2 s$ electrons were treated as valence electrons, and core-electron interactions were modeled using the projector-augmented wave method [? ]. The Hubbard $U$ correction as derived by Dudarev et al. [? ] was applied to the

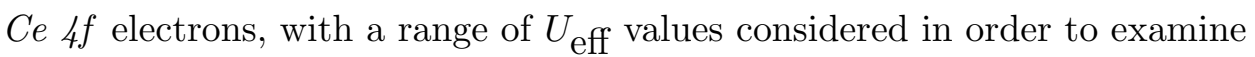
the effects of $f$-electron localization on the present results [? ? ]. Electronic wavefunctions were expanded in plane waves with a kinetic-energy cutoff of $400 \mathrm{eV}$. Reciprocal-space summations were performed at the $\Gamma-$ point. Atomistic structures were constructed in a calculation supercell to which periodic boundary conditions are applied. Each supercell contains a nanoparticle structure surrounded by vacuum (empty space). The minimum separation between CNPs in neighboring supercells (due to periodic boundary conditions) is $\sim 15 \AA$.

All results detailed below are for fully "relaxed" atomic structures. That is, initial atomic geometries are evolved according to the calculated interatomic forces seeking a minimum energy (instantaneous ground state) configuration. This is functionally equivalent to minimizing the instantaneous 
forces, as forces are simply the set of derivatives of the total energy with respect to each atomic degree of freedom. For the present calculations all considered structures were relaxed without symmetry constraints until maximum residual atomic forces were less than $0.04 \mathrm{eV} / \AA$ and total energies were converged to within $10^{-4} \mathrm{eV} /$ atom.

\subsection{Initial atomic geometries}

Initial nanoparticle structures were constructed from cubic fluorite (space group Fm $\overline{3} \mathrm{~m}$ ) FCC unit cells containing 1 Ce atom at the unit cell corners (where all eight cube corners are identical due to symmetry) and 3 Ce atoms at the center of the unit cell faces (where each opposite pair of faces are identical due to symmetry). Eight $\mathrm{O}$ atoms are located at the FCC tetrahedral interstitial sites, giving $4 \mathrm{Ce}$ atoms and $8 \mathrm{O}$ atoms in each unit cell (see Figure 2a). Initial CNP structures with various sizes and shapes were constructed by repeating this unit cell in three dimensions (see, e.g., Figure 2b).

As we are focusing on the stability of O-terminated CNPs as a function of surface O concentration, we take ideal, O-saturated CNPs as structural baselines. That is, initial atomic geometries for CNPs with varying $\mathrm{O}$ concentrations are constructed as modifications of the appropriately shaped baseline (fully O-terminated) structure. Examples of these baseline structures are shown in Figures 2c-2f. In these structures all $\mathrm{O}$ lattice sites are occupied, including those that are symmetrically equivalent (e.g., O lattice sites on opposite facets or on corners other than the origin). In contrast, stoichiometric structures would have only the symmetrically distinct $\mathrm{O}$ lattice sites occupied. Occupying all O lattice sites (as is shown in Figures 2c-2f) results in super-stoichiometric structures. For example, the ideal (bulk-like) $\sim 1 \mathrm{~nm}\{100\}$-terminated CNPs in Figures 2c and 2d with all $\mathrm{O}$ lattice 
Table 1: Calculated and experimental data used to determine $\mu_{O}^{*}$. Experimental enthalpies of formation are taken from reference [? ]. See text for details.

\begin{tabular}{c|c|c|c|c|c|c|c}
\hline \hline Calculation & $\mathrm{E}\left(\mathrm{CeO}_{2}\right)$ & $\mathrm{E}\left(\mathrm{Ce}_{2} \mathrm{O}_{3}\right)$ & $\mathrm{E}(\mathrm{Ce})$ & $\mathrm{E}\left(\mathrm{O}_{2}\right)$ & $\Delta \mathrm{E}$ & $\Delta \mathrm{H}\left(\mathrm{CeO}_{2}\right)$ & $\Delta \mathrm{H}\left(\mathrm{Ce}_{2} \mathrm{O}_{3}\right)$ \\
$\mathrm{LDA}(\mathrm{np}, \mathrm{U}=5 \mathrm{eV})$ & -27.0182 & -43.4676 & -4.8469 & -9.5548 & 0.77 & -11.8465 & -18.2866 \\
$\mathrm{GGA}(\mathrm{np}, \mathrm{U}=3 \mathrm{eV})$ & -24.6554 & -40.3456 & -4.6046 & -8.7796 & -0.33 & -11.6012 & -18.4620 \\
\hline Experiment & - & - & - & - & - & -11.3010 & -18.6530 \\
\hline \hline
\end{tabular}

sites occupied have the composition of $\mathrm{Ce}_{32} \mathrm{O}_{121}$-with 94 out of total 121 $\mathrm{O}$ atoms at the CNP surface. Straightforward mathematical rules for the number of $\mathrm{O}$ and $\mathrm{Ce}$ lattice sites in CNPs of various shapes and arbitrary sizes can be developed, and are reported in detail in Appendix A.

In searching for low-energy atomic configurations with varying numbers of $\mathrm{O}$ atoms at $\mathrm{CNP}$ surfaces we have considered a wide range input $\mathrm{O}$ configurations beyond the baseline initial structures. In constructing input structures for relaxation calculations, $\mathrm{O}$ atoms have been placed in various configurations at CNP surfaces, including at high-symmetry sites (e.g., atop Ce atoms, or bridging two or three Ce atoms), as well as in positions selected to mimic or perturb subsequent relaxation into structures involving pairs or triples of $\mathrm{O}$ atoms. While it is not feasible to consider all possible initial arrangements of surface $\mathrm{O}$ atoms, we have demonstrated the robustness of our present results with respect to input $\mathrm{O}$ surface configurations by testing whether the considered structures relaxed into local or global energy minima using finite temperature molecular dynamics calculations on a subset of relaxed structures. In all cases structures resulting from MD calculations were quantitatively similar to structures resulting from relaxation only, indicating that the relaxed configurations are at worst deep local minima. 


\subsection{1. $\{100\}$-terminated cubic CNPs}

In the following we explicitly consider cubic $\{100\}$-terminated CNPs, in addition to previously considered octahedral and cuboctahedral structures. $\{100\}$-terminated CNPs are considered because such shapes have been observed experimentally (see, e.g., Figure 3). In addition, previous experimental efforts have found that $\{100\}$ facets exhibit greater catalytic activity then other ceria surfaces, and represent the dominant active surface in a range of nanometer scale geometries (e.g., nanoplates, nanorods, and nanowires) [? ? ? ? ? ]. This is supported by previous computational work showing that $\{100\}$ surfaces have lower formation energies of O-vacancies, and (following the "four-step" hypothesis) are therefore expected to have greater activity [? ? ? ]. In the present work, we consider $\sim 1 \mathrm{~nm}$ cubic CNPs that are $2 \times 2 \times 2$ repeats of the bulk $\mathrm{CeO}_{2}$ unit cell (see Figures $2 \mathrm{c}$ and $2 \mathrm{~d}$ ). For

cubic CNPs, its six surfaces are terminated by $\{100\}$ ceria facets. To ensure that no interactions occur between any calculated CNP and minimize the computation cost, the size of the supercell is set to $2.7 \mathrm{~nm} \times 2.7 \mathrm{~nm} \times$ $2.7 \mathrm{~nm}$ so that neighboring CNPs are separated by at least $1.5 \mathrm{~nm}$ vacuum space when periodic boundary condition is applied (see Figure 2c). The ideal bulk-like $\sim 1 \mathrm{~nm}$ cubic CNP has the composition of $\mathrm{Ce}_{32} \mathrm{O}_{121}$, with 32 Ce atoms (yellow balls), 27 bulk O atoms (purple balls) and 94 surface $\mathrm{O}$ atoms (blue balls).

\subsection{2. $\{111\}$-terminated octahedral and cuboctahedral CNPs}

For octahedral CNPs, all eight surfaces are terminated by $\{111\}$ ceria facets. The $\sim 1 \mathrm{~nm}$ octahedral CNP is obtained directly as eight intersecting $\{111\}$ bulk cuts through a $4 \times 4 \times 4$ set of bulk $\mathrm{CeO}_{2}$ unit cells. The eight $\{111\}$ cuts then give a bulk-terminated CNP with a composition of 
$\mathrm{Ce}_{44} \mathrm{O}_{160}$. Of the $160 \mathrm{O}$ atoms, 80 are fully-coordinated bulk $\mathrm{O}$ atoms and 80 are under-coordinated surface $\mathrm{O}$ atoms (see Figures $2 \mathrm{e}$ and $2 \mathrm{f}$ ). As above, the supercell for $\sim 1 \mathrm{~nm}$ octahedral CNPs is constructed to have an intraparticle seperation of at least $1.5 \mathrm{~nm}$, and therefore is assigned a net size of $3.8 \mathrm{~nm} \times 3.8 \mathrm{~nm} \times 3.8 \mathrm{~nm}$ (see Figure $2 \mathrm{e}$ ). Cuboctahedral $\sim 1 \mathrm{~nm}$ CNPs are modifications of octahedral CNPs and are constructed (following reference [? ]) by deleting all 80 surface $\mathrm{O}$ atoms as well as an arbitrary selection of four of the six corner Ce atoms. This gives a CNP with an enforced stoichiometric composition of $\mathrm{Ce}_{40} \mathrm{O}_{80}$.

3.3. Excess energy of formation as a function of CNP size, shape and composition

To compare energies of CNPs with different compositions (and/or shapes, surface configurations, etc.), we must define an energy of formation with a common reference. Here we focus on the relative energy of CNPs with varying numbers of surface $\mathrm{O}$ atoms. We therefore define an excess energy of formation term, $\Delta E_{f}^{C N P}\left(n_{O}\right)$, as referenced to the energy of a reduced $\sim 1 \mathrm{~nm}$ CNP. Changes in the excess energy of formation therefore indicate whether a particular CNP structure is more or less stable (has lower or higher energy) than the reference (a reduced) CNP structure.

$$
\Delta E_{f}^{C N P}\left(n_{O}\right) \equiv E_{t o t}^{C N P}-E_{t o t}^{r e f}-\left(n_{O}-n_{O}^{r e f}\right) \mu_{O}
$$

Here $n_{O}$ is the number of $\mathrm{O}$ atoms in the CNP of interest, and $n_{O}^{r e f}$ is the number of $\mathrm{O}$ atoms in the reference CNP. $\mu_{O}$ is the chemical potential of $\mathrm{O}$ (the chemical potential of $\mathrm{O}_{2}$ molecule per $\mathrm{O}$ atom). To facilitate comparisons between CNPs of various sizes and shapes, we further write 
this excess energy of formation per Ce atom

$$
\Delta e_{f}^{C N P}\left(n_{O}\right) \equiv \frac{\Delta E_{f}^{C N P}}{n_{C e}}
$$

Equations 1 and 2 are direct measures of the relative energy of different CNP configurations. The selection of the reference CNP is arbitrary, and simply serves to set the zero of the energy scale. As a link to previous calculations, which have primarily focused on partially reduced CNPs, we select the reduced cubic $\mathrm{CNP}, \mathrm{Ce}_{32} \mathrm{O}_{57}$, as the reference CNP structure for the present calculations of the excess energies of formation. This structure is constructed from the fully O-saturated baseline cubic CNP $\left(\mathrm{Ce}_{32} \mathrm{O}_{121}\right.$, see Figure 2d) by deleting all corner and edge $\mathrm{O}$ atoms, as well as 4 face $\mathrm{O}$ atoms near each CNP corner on every $\{100\}$ facet.

The chemical potential of $\mathrm{O}$ appearing in Eq. 1 characterizes the energy cost of additional $\mathrm{O}$ atoms, and allows the comparison of energies for structures with varying numbers of $\mathrm{O}$ atoms. The energy cost of $\mathrm{O}$ atoms in real systems is determined by the state of available $\mathrm{O}$ atoms from the environment surrounding the CNP. For the present calculations, we take pure $\mathrm{O}_{2}$ gas as the environmental reference state. This is a constraint on real systems, where other atomic or molecular species are almost invariably present, but defines a tractable scope for the present work and represents a useful starting point for systematic consideration of CNP surface configurations in open systems. Note that chemical potential is physically related to the partial pressure, e.g. $p_{\mathrm{O}_{2}}$, and more negative values of $\mu_{O}$ correspond to lower $p_{\mathrm{O}_{2}}[?]$. Therefore the largest energy cost $\left(\mu_{O}=\frac{1}{2} E_{\mathrm{O}_{2}}\right.$, where $E_{\mathrm{O}_{2}}$ is the calculated ground state energy of a single $\mathrm{O}_{2}$ molecule) for the addition of an $\mathrm{O}$ atom occurs in the dilute gas limit, and this cost represents the most negative physically-realizable value possible for $\mu_{O}$. 
DFT calculations of metal oxide oxidation energies are known to exhibit two separate errors: an error in the $\mathrm{O}_{2}$ intermolecular binding energy and an error in the change in the energy upon transfer of electrons between $\mathrm{O}$ $2 p$ orbitals and metal $d$ or $f$ orbitals [? ? ]. In addition, the spin state of $\mathrm{O}$ atoms also appreciably affects DFT calculated energies of $\mathrm{O}_{2}$ molecules. By fitting calculated oxidation energies to experimentally determined enthalpies of formation, a net correction to the chemical potential of $\mathrm{O}$ that addresses all of these issues has been calculated for a range of transition metal oxides [? ]. Here we apply an analogous correction by referencing experimental enthalpies of formation for $\mathrm{CeO}_{2}$ and $\mathrm{Ce}_{2} \mathrm{O}_{3}$ [? ] to determine a corrected value for the chemical potential of $\mathrm{O}, \mu_{O}^{*}$. Choosing $\mu_{O} \equiv \mu_{O}^{*}$ therefore connects DFT calculations of CNP energies to experimentally-relevant reference values of the chemical potential of $\mathrm{O}$ and overcomes computational challenges in directly calculating such values. In other words, correcting DFT calculated reference energies of $\mathrm{O}$ by fitting experimental enthalpies of formation for bulk cerium oxides gives a best-case representation of the energy cost of $\mathrm{O}$ atoms from real O-containing environments in this study.

Following Wang et al. [? ], the appropriate value of $\mu_{O}^{*}$ is found by first calculating the energy of formation for $\mathrm{O}_{2}\left(E\left(O_{2}\right)\right)$ with the same calculation methodology and settings as those used to calculate CNP energies. At the same time, the energies of formation for bulk $\mathrm{Ce}(E(C e))$, bulk $\mathrm{CeO}_{2}$ $\left(E\left(\mathrm{CeO}_{2}\right)\right)$ and bulk $\mathrm{Ce}_{2} \mathrm{O}_{3}\left(E\left(\mathrm{Ce}_{2} \mathrm{O}_{3}\right)\right)$ are calculated. The calculated enthalpies of formation for bulk $\mathrm{CeO}_{2}$ and $\mathrm{Ce}_{2} \mathrm{O}_{3}$ are then [? ]:

$$
\begin{gathered}
\Delta H\left(\mathrm{CeO}_{2}\right)=E\left(\mathrm{CeO}_{2}\right)-E(\mathrm{Ce})-E\left(\mathrm{O}_{2}\right) \\
\Delta H\left(\mathrm{Ce}_{2} \mathrm{O}_{3}\right)=\frac{2 E\left(\mathrm{Ce}_{2} \mathrm{O}_{3}\right)-3 E\left(\mathrm{O}_{2}\right)}{2}-2 E(\mathrm{Ce})
\end{gathered}
$$

A correction $\Delta E$ is applied to $E\left(O_{2}\right)$ so that the root mean square variation 
of the calculated enthalpies of formation for bulk $\mathrm{CeO}_{2}$ and $\mathrm{Ce}_{2} \mathrm{O}_{3}$ from experimentally-measured values [? ] is minimized. Note that energies of formation for $\mathrm{O}_{2}$, bulk $\mathrm{Ce}$, bulk $\mathrm{CeO}_{2}$ and bulk $\mathrm{Ce}_{2} \mathrm{O}_{3}$ were calculated using both LDA $+U$ and GGA $+U$ approaches $\left(U_{\text {eff }}=5 \mathrm{eV}\right.$ for LDA and $U_{\text {eff }}=3 \mathrm{eV}$ for GGA). All the relevant data are summarized in Table 1. The corrected chemical potential of $\mathrm{O}$ for gaseous $\mathrm{O}_{2}, \mu_{O}^{*}$, is evaluated via $\mu_{O}^{*}=\frac{1}{2}\left(E_{O_{2}}-\Delta E\right)$ as $-5.16 \mathrm{eV}$ for LDA and $-4.23 \mathrm{eV}$ for GGA.

\section{Results}

We now turn to the question of determining the stable structure of CNP surfaces when the surface $\mathrm{O}$ concentration and configuration are allowed to vary from that of the underlying bulk. Beginning with the initial atomic structures described above, we have relaxed the atomic configurations of cubic $\{100\}$ CNPs with diameters of $\sim 1-1.5 \mathrm{~nm}$ and a range of surface $\mathrm{O}$ concentrations as well as octahedral and cuboctahedral $\{111\}$ CNPs with diameters of $\sim 1 \mathrm{~nm}$ and a range of surface $\mathrm{O}$ concentrations. Using Equations 1 and 2, the excess energy of formation per Ce atom has been calculated for each CNP structure. Figure 4 shows the results of these calculations in the O-lean limit (that is, at the corrected chemical potential of $\mathrm{O}, \mu_{O}^{*}$ ). For all considered CNP shapes, Figure 4 shows that small CNPs can reduce their excess energy of formation by increasing the $\mathrm{O}$ concentration at their surfaces. Because the chemical potential of $\mathrm{O}$ only increases for increasing partial pressures of $\mathrm{O}_{2}$ - that is, the energy cost of $\mathrm{O}$ atoms decreases as the environment moves from the O-lean limit towards the O-rich limitFigure 4 demonstrates that oxidized CNPs $\left(\mathrm{CeO}_{2+x}\right)$ are stable in both O-lean $\left(\mu_{O} \equiv \mu_{O}^{*}=-5.16 \mathrm{eV}\right.$ for LDA and $-4.23 \mathrm{eV}$ for GGA, excess ener- 
gies plotted in Figure 4) and O-rich $\left(\mu_{O}>-5.16 \mathrm{eV}\right.$ for LDA and $-4.23 \mathrm{eV}$ for GGA) environments. In the latter case, O-enriched structures are even more stable with respect to reduced CNPs, as the cost of the additional $\mathrm{O}$ atoms is less. Note that both LDA and GGA results exhibit the same trend in excess energy of formation as a function of $n_{O} / n_{C e}$ for all considered shapes. In addition, while Figure 4 only shows results for $U_{\text {eff }}=5$ and $3 \mathrm{eV}$ (for LDA and GGA, respectively), calculations for $U_{\text {eff }}=1$ and $3 \mathrm{eV}$ (LDA) and $U_{\text {eff }}=1$ and $5 \mathrm{eV}(\mathrm{GGA})$ also reveal the same trend: that increased surface $\mathrm{O}$ concentration reduces CNP excess energy of formation.

Addressing the possible special case of exactly stoichiometric CNPs, the excess energy of formation for the $\sim 1 \mathrm{~nm}$ cuboctahedral CNP with composition $\mathrm{Ce}_{40} \mathrm{O}_{80}$ is explicitly included in Figure 4. While the stoichiometric cuboctahedral CNP does exhibit a local minimum in energy with respect to O composition, numerous oxidized CNPs, including oxidized cuboctahedral CNPs of the same shape and size, have lower excess energies of formation. That is, even relative to exactly stoichiometric CNPs, oxidized CNPs with enhanced surface $\mathrm{O}$ concentrations have lower excess energies of formation. These results demonstrate that the thermodynamically stable configuration that a sufficiently small CNP begins in and returns to before and after catalyzing a redox reaction is not stoichiometric, rather the surfaces of thermodynamically stable CNPs that are sufficiently small are O-enriched.

Figure 5 shows the atomic structures of representative relaxed cubic, octehedral and cuboctahedral CNPs with reduced (top left panel for each shape) and oxidized net compositions (top right and bottom panels). Excess energies of formation for these structures are all included in Figure 4. It is apparent that stoichiometrically "excess" O atoms in oxidized structures appear at the CNP corners, edges and $\{100\}$ facets in the form of either 
Table 2: Properties of $\mathrm{O}$ pairs and triples in representative oxidized CNPs.

\begin{tabular}{c|c|c|c|c}
\hline \hline System & Bond length $(\AA)$ & Bond angle $\left(^{\circ}\right)$ & Bader charge & Molecule Assignment \\
\hline \multirow{5}{*}{$\mathrm{Ce}_{32} \mathrm{O}_{179}$} & $1.24 \pm 0.00$ & - & $-0.18 \pm 0.02$ & $\mathrm{O}_{2}$ \\
& $1.31 \pm 0.03$ & - & $-0.53 \pm 0.16$ & $\mathrm{O}_{2}^{-}$ \\
& $1.42 \pm 0.02$ & - & $-1.07 \pm 0.05$ & $\mathrm{O}_{2}^{2-}$ \\
& $1.33 \pm 0.02,1.30 \pm 0.01$ & $111.96 \pm 0.38$ & $-0.39 \pm 0.04$ & $\mathrm{O}_{3}$ \\
& $1.42 \pm 0.03,1.40 \pm 0.02$ & $105.36 \pm 1.24$ & $-1.11 \pm 0.11$ & $\mathrm{O}_{3}^{-}$ \\
\hline \multirow{2}{*}{$\mathrm{Ce}_{44} \mathrm{O}_{152}$} & $1.25 \pm 0.00$ & - & $-0.24 \pm 0.03$ & $\mathrm{O}_{3}^{2-}$ \\
\hline & $1.28 \pm 0.01$ & - & $-0.39 \pm 0.03$ & $\mathrm{O}_{2}$ \\
& $1.25 \pm 0.00$ & - & $-0.19 \pm 0.02$ & $\mathrm{O}_{2}^{-}$ \\
\hline \multirow{2}{*}{$\mathrm{Ce}_{40} \mathrm{O}_{152}$} & $1.27 \pm 0.00$ & - & $-0.35 \pm 0.01$ & $\mathrm{O}_{2}$ \\
& $1.42 \pm 0.02,1.30 \pm 0.01$ & $110.97 \pm 0.70$ & $-0.76 \pm 0.03$ & $\mathrm{O}_{2}^{-}$ \\
\hline \hline
\end{tabular}

$\mathrm{O}$ pairs or $\mathrm{O}$ triples. In Figure 5, Ce atoms are yellow, single (lattice) $\mathrm{O}$ atoms are purple, and $\mathrm{O}$ atoms in pairs (triples) are indicated with warm (cool) colors. The relaxed net oxidized CNP structures represent substantial rearrangements of $\mathrm{O}$ atoms relative to the initial (input) structures, and $\mathrm{O}$ pairs and triples formed spontaneously as a result of structural relaxations minimizing the total CNP energy for all considered (oxidized) input structures. Only individual (crystalline) O lattice atoms appear in reduced CNP structures, while O pairs and triples only appear in oxidized CNP structures. That is, while the assumption of bulk-like surface terminations is valid for reduced $\sim 1-1.5 \mathrm{~nm}$ CNPs, thermodynamically stable $\sim 1-1.5 \mathrm{~nm}$ CNPs in real O-containing environments are oxidized (see Figure 4) and do not exhibit bulk-like arrangements of surface $\mathrm{O}$ atoms.

For every O pair or triple present on a relaxed (oxidized) CNP, the distance between at least one $\mathrm{O}$ atom in each pair or triple and a Ce atom is within the range of $\mathrm{Ce}-\mathrm{O}$ bond lengths observed in bulk $\mathrm{CeO}_{2}$ or $\mathrm{Ce}_{2} \mathrm{O}_{3}$ 
(that is, between 2.34 and $2.67 \AA$ ). This is highlighted in the lower panels of Figure 5 which show the same view of each CNP shape as presented in the upper right panels, but exposes the $\mathrm{O}-\mathrm{Ce}$ and $\mathrm{O}-\mathrm{O}$ binding networks. In these images bonds are drawn for Ce-O distances less than $2.5 \AA$ And for O-O distances less than $1.5 \AA$.

The $\mathrm{O}-\mathrm{O}$ binding configurations of $\mathrm{O}$ pairs and triples are consistent with the bond lengths and angles of isolated $\mathrm{O}_{2}^{q}$ and $\mathrm{O}_{3}^{q}$ molecules for $q=0$, -1 or -2 . This is shown in Table 2, which reports calculated $\mathrm{O}-\mathrm{O}$ bond lengths, angles, and local charges (assigned per atom following the method of Bader [? ], which provides a qualitative assignment of electrons to particular atoms) for O pairs and triples on oxidized CNPs. Based on comparisons to the Bader charge on lattice $\mathrm{O}$ atoms in bulk $\mathrm{CeO}_{2}(-1.18)$ which are formally $\mathrm{O}^{\mathrm{II}}$ and the calculated bond lengths and angles of isolated $\mathrm{O}_{x}^{q}$ molecules, we conclude that the excess $\mathrm{O}$ atoms present on oxidized CNPs are, in fact, variously charged $\mathrm{O}_{x}^{q}$ groups chemically adsorbed at CNP corners, edges and $\{100\}$ facets.

For all studied oxidized CNP configurations, the removal of all $\mathrm{O}$ atoms present after relaxation in the form of adsorbed molecular $O_{x}^{q}$ groups leaves net reduced CNPs. This suggests that low-energy $\sim 1-1.5 \mathrm{~nm}$ CNP configurations (all of which are oxidized, see Figure 4) can be considered reduced CNPs passivated with surface adsorbed molecular $\mathrm{O}_{x}^{q}$ groups. This picture is directly supported by a recent analysis by Preda et al. [? ], of the energetics and electronic effects of the adsorption a single $\mathrm{O}_{2}$ molecule on the surface of reduced CNPs and is consistent with previous experimental studies [?] reporting spectroscopic evidence for the adsorption of superoxo and peroxo species on reduced ceria surfaces.

Since the $C e^{\mathrm{III}}-\mathrm{Ce}^{\mathrm{IV}}$ redox couple is the engine driving the catalytic 
properties of CNPs, the oxidation states of Ce cations in relaxed CNPs have also been probed. As above, atomic charges are calculated via the Bader procedure [? ]. Ce atoms in reduced CNPs exhibit a range of charge states generally intermediate between the charge states of $\mathrm{Ce}^{\mathrm{III}}$ and $\mathrm{Ce}^{\mathrm{IV}}$ as determined from calculations of bulk $\mathrm{Ce}_{2} \mathrm{O}_{3}$ and $\mathrm{CeO}_{2}$. Lower Hubbard $U$ parameters (which reduce the $U$ energy penalty against extended states) decrease the net positive charge on each Ce atom, but do not change the qualitative distribution of charges. Increased $\mathrm{O}$ concentration (from reduced $\mathrm{CeO}_{2-x}$ to super-oxidized $\mathrm{CeO}_{2+x}$ structures) shifts the charge distribution of Ce atoms in considered CNP structures towards $\mathrm{Ce}^{\mathrm{IV}}$, but produces no Ce atoms with net charges significantly greater than $\mathrm{Ce}^{\mathrm{IV}}$. Table 3 summarizes calculated average Bader charges of Ce atoms in a set of cubic CNP structures as an example, and their oxidation states can be analyzed by comparing to the calculated average Bader charge of Ce atoms in bulk $\mathrm{CeO}_{2}$ and $\mathrm{Ce}_{2} \mathrm{O}_{3}$.

We are now in a position to comment on why the formation of $\mathrm{O}_{x}^{q}$ surface groups substantially reduces the excess energy of formation for sufficiently small CNPs. As a rare earth oxide with negative enthalpy of formation (see Table 1), we expect $\mathrm{CeO}_{2}$ to prefer O-terminated surfaces in O-containing environments. This is supported by STM studies of the $\{111\} \mathrm{CeO}_{2}$ surface [? ] and atomistic calculations [? ? ]. In fact, if this were not so, Ce metal would be expected to be more stable relative to ceria in $\mathrm{O}$ environments. While the ceria $\{111\}$ surface has a stoichiometric O-terminated structure, O-termination of CNP corners, edges and $\{100\}$ facets requires a supersaturation of $\mathrm{O}$ atoms. Therefore, for sufficiently small CNPs, O-termination of CNP corners, edges and $\{100\}$ facets implies that the overall CNP be super-oxidized $\left(\mathrm{CeO}_{2+x}\right)$. In turn, excess $\mathrm{O}$ implies either the presence of 
Table 3: Average Bader charge of Ce atoms in cubic CNPs with different O concentrations obtained from LDA $+\mathrm{U}$ calculations with $U=5$.

\begin{tabular}{c|c|c}
\hline \hline $\begin{array}{c}\text { CNPs with different } \\
\text { O concentrations }\end{array}$ & $\begin{array}{c}\text { Average Bader charge } \\
\text { of Ce atoms }\end{array}$ & $\begin{array}{c}\text { CNP } \\
\text { type }\end{array}$ \\
\hline $\mathrm{Ce}_{32} \mathrm{O}_{59}$ & $2.185 \pm 0.067$ & reduced \\
$\mathrm{Ce}_{32} \mathrm{O}_{89}$ & $2.291 \pm 0.032$ & oxidized \\
$\mathrm{Ce}_{32} \mathrm{O}_{101}$ & $2.302 \pm 0.039$ & oxidized \\
$\mathrm{Ce}_{32} \mathrm{O}_{121}$ & $2.309 \pm 0.038$ & oxidized \\
$\mathrm{Ce}_{32} \mathrm{O}_{147}$ & $2.314 \pm 0.033$ & oxidized \\
$\mathrm{Ce}_{32} \mathrm{O}_{169}$ & $2.321 \pm 0.028$ & oxidized \\
$\mathrm{Ce}_{32} \mathrm{O}_{179}$ & $2.331 \pm 0.028$ & oxidized \\
\hline bulk $\mathrm{Ce}_{2} \mathrm{O}_{3}$ & $\sim 1.98$ & - \\
bulk $\mathrm{CeO}_{2}$ & $\sim 2.35$ & - \\
\hline \hline
\end{tabular}

Ce atoms oxidized beyond $\mathrm{Ce}^{\mathrm{IV}}$ — a situation not observed in the present study - or, that not all $\mathrm{O}$ atoms are doubly reduced. This last is the case as observed here, as highlighted in Table 2. In a structure with excess $\mathrm{O}$ atoms that have not relaxed to form $\mathrm{O}_{x}^{q}$ groups, individual $\mathrm{O}$ atoms that are not doubly reduced (that is $\mathrm{O}_{1}^{-1}$ anions) are most likely to be undercoordinated $\mathrm{O}$ atoms at the corners, edges or $\{100\}$ facets of CNPs. An under-coordinated corner/edge/facet $\mathrm{O}$ atom with unfilled $p$ orbitals (that is, not doubly reduced) is highly reactive. Interaction with other $\mathrm{O}$ atoms allows reactive $\mathrm{O}_{1}^{-1}$ atoms to form more stable molecular $\mathrm{O}_{2} / \mathrm{O}_{2}^{-} / \mathrm{O}_{2}^{2-}$ and $\mathrm{O}_{3} / \mathrm{O}_{3}^{-} / \mathrm{O}_{3}^{2-}$ groups. These then appear as adsorbed $\mathrm{O}_{x}^{q}$ groups on CNP corners, edges and $\{100\}$ facets.

For CNPs with bulk portions that are stoichiometric $\mathrm{CeO}_{2}$, this effect 
(that excess $\mathrm{O}$ implies $\mathrm{Ce}$ atoms oxidized beyond $\mathrm{Ce}^{\mathrm{IV}}$ or $\mathrm{O}$ atoms that are not doubly reduced) would be present at all particle sizes. But as bulk ceria prefers a partially reduced form (that is, some fraction of all Ce atoms are Ce $\mathrm{III}^{\mathrm{II}}$ at finite temperature) [? ], large enough particles (e.g., particles with sufficient internal bulk volume) can sustain the additional $\mathrm{O}$ atoms necessary to yield O-terminated surfaces while remaining net reduced $\left(\mathrm{CeO}_{2-x}\right)$ - and hence not requiring Ce atoms oxidized beyond $\mathrm{Ce}^{\mathrm{IV}}$ or $\mathrm{O}$ atoms that are not doubly reduced. For even larger ceria samples, sufficient O-vacancies exist in the bulk that, via diffusion, an equilibrium concentration of surface Ovacancies will appear at finite temperature. In this way, the present picture is completely consistent with previous calculations and experiments showing that large ceria particles (and bulk ceria) are partially reduced [? ].

Furthermore, by assuming a net bulk O-vacancy concentration of $5 \%$ [? ? ] and leveraging geometrical rules for the number of $\mathrm{Ce}$ and $\mathrm{O}$ atoms present in CNPs of various shapes (see Appendix A), the net Ce/O ratio can be estimated as a function of the size for O-terminated cubic and octahedral CNPs. Figure 6 shows that the transition from larger, reduced (and therefore bulk-terminated) CNPs to smaller, $\mathrm{O}_{x}^{q}$ group-terminated CNPs occurs at critical sizes between 8 and $35 \mathrm{~nm}$, depending on the CNP geometry. These critical sizes are the result of two competing effects-bulk thermodynamic effects driving net reduction of ceria, and surface configurations driving net oxidation of ceria. Decreases (or increases) in the net O-vacancy concentration do not alter this qualitative result, but rather lead to small increases (or decreases) in the critical size (e.g., from 25 to $15 \mathrm{~nm}$ for $\{100\}$-terminated CNPs after a decrease in O-vacancy concentration to $3 \%)$. 


\section{Discussion and conclusions}

Ceria and ceria-based materials are widely applied as redox catalysts or catalyst supports and have been extensively studied. The established picture of catalysis (or catalysis support) by ceria is based on assumptions of bulklike ceria surface structures. While these assumptions are well-supported for large ceria systems (e.g., semi-infinite ceria surfaces), we show here that, below a critical size, CNPs in pure O environments do not exhibit bulk-like surface terminations. Instead, they exhibit stable surface reconstructions consisting of $\mathrm{O}_{x}^{q}(-2 \leq \mathrm{q} \leq 0, \mathrm{x}=2,3)$ groups chemically adsorbed at CNP corners, edges, and $\{100\}$ facets. The formation of $\mathrm{O}_{x}^{q}$ groups reduces the energy of small CNPs by increasing the coordination of near surface $\mathrm{Ce}$ cations, while allowing for the formation of low-energy surface $\mathrm{O}_{x}^{q}$ groups in a range of oxidation states.

The existence of this balance (increased cation coordination stabilized by the formation of $\mathrm{O}_{x}^{q}$ groups in various oxidation states) is necessitated by the non-stoichiometry of CNP corners, edges and (as considered here) $\{100\}$ facets. Assuming that no $\mathrm{O}$ vacancies are present within a CNP, non-stoichiometric corners, edges, and/or facets imply either that undercoordinated Ce cations are exposed at CNP corners, edges and/or facets, or that (in $\mathrm{O}$ environments) excess $\mathrm{O}$ anions are present on CNPs. If CNPs are O-terminated then either not all $\mathrm{O}$ anions are doubly reduced, or some $\mathrm{Ce}$ cations are oxidized beyond $\mathrm{Ce}$ IV . Here we show that for experimentallyrelevant $\mathrm{O}$ chemical potentials, $\sim 1-1.5 \mathrm{~nm}$ CNPs have minimum energies of formation when they are O-terminated with $\mathrm{O}$ anion groups that are not doubly reduced. O that is not doubly reduced is more stable in the form of molecular $O_{x}^{q}$ groups, and, therefore, thermodynamically stable configu- 
rations of $\sim 1-1.5 \mathrm{~nm}$ CNPs in $\mathrm{O}$ environments are terminated with bound $\mathrm{O}_{x}^{q}$ groups.

As bulk ceria is known to exhibit finite concentrations of lattice $\mathrm{O}$ vacancies, there exists a critical size beyond which all near-surface Ce cations can be fully $\mathrm{O}$ coordinated without requiring the presence of stoichiometrically excess $\mathrm{O}$ atoms. That is, for sufficiently large CNPs, enough bulk vacancies are present to offset the surface $\mathrm{O}$ atoms required to fully coordinate nearsurface Ce cations. This leads to a shape-dependent critical size (estimated here as between 8 and $35 \mathrm{~nm}$ for $\{100\}$ - and $\{111\}$-terminated CNPs) for a transition from atomic-O (bulk-like) surface terminations to $\mathrm{O}_{x}^{q}$ surface terminations. For this reason, large ceria systems are expected to exhibit bulk-like O-terminations - as observed [? ] —while small nanoceria particles are expected to exhibit a completely different class of surface terminations characterized by the formation of surface anion groups that need not be doubly oxidized.

It should be noted that the qualitative result that stoichiometrically excess surface $\mathrm{O}$ atoms form $\mathrm{O}_{x}^{q}$ groups at $\mathrm{CNP}$ surfaces is very robust with respect to the input configuration of surface $\mathrm{O}$ atoms. While variations in the arrangement of $\mathrm{O}_{x}^{q}$ groups themselves are possible (particularly at finite temperature) and have not been considered in detail here, such variations will not alter the core finding that $\mathrm{O}_{x}^{q}$ groups are expected be present on sufficiently small CNPs. In addition, we note that the anion groups bound to near-surface Ce cations need not be $\mathrm{O}_{x}^{q}$ groups. In fact, extending the picture introduced above of stable, oxidized CNPs as reduced CNPs passivated with chemically adsorbed anion groups, we note that the adsorbed surface groups themselves represent the interface between the CNP and its environment. Therefore any environmental anion group could serve to sat- 
isfy near-surface Ce coordination requirements. In the present calculations, we have considered only pure $\mathrm{O}$ environments, but CNPs are widely synthesized and applied in either liquid or gas environments containing water. In that case, surface-adsorbed -OH groups will substitute for $\mathrm{O}_{x}^{q}$ groups, as we discuss in detail elsewhere [? ]. In environments containing other species, any available anionic groups could potentially be directly bound at the surfaces of sufficiently small CNPs.

That the intrinsic nature of CNP surfaces - for CNPs below a critical size - is not bulk-like, but rather consists of environmentally-determined configurations of variously reduced surface anion groups is a key insight into catalysis by small CNPs. In CNP catalysis, the reactants and products of any catalyzed reaction will be present as part of the environment surrounding the CNP. In fact, many of the most important reactions in which CNP catalysts are applied have $\mathrm{O}_{x}^{q}$ and/or - $\mathrm{OH}$ groups as important reactants or products. As sufficiently small CNPs will intrinsically bind these groups directly, we must consider the possibility of catalytic mechanisms at small CNPs that do not involve the formation and/or migration of bulk Ovacancies (that is, mechanisms that differ from the accepted picture of ceria redox catalysis). Hence, for instance, reaction rates for catalytic oxidation of $\mathrm{CO}$ or NO over small CNPs may not be limited by the thermodynamics and kinetics of bulk and surface O-vacancies, but rather by the direct uptake and release of variously oxidized molecular anion groups at CNP corners, edges, and non-stiochiometric (e.g., $\{100\})$ facets.

This last point is dramatically highlighted by numerous reports in the literature of the enhanced catalytic properties of $\{100\}$ facets in small CNPs [? ? ? ? ? ? ? ? ? ]. The present results provide an elegant explanation for these observations: in contrast to stiochiometric $\{111\}$ facets, non- 
stiochiometric $\{100\}$ facets on sufficiently small CNPs intrinsically adsorb anionic reactants in various oxidation states. Therefore, redox reactions catalyzed at $\{100\}$ CNP facets need not wait for the thermally driven creation of lattice $\mathrm{O}$ vacancies.

As a final note, the ability to tune what sufficiently small CNPs "look like" during catalysis - that is, the ability to tune the anion surface group "shell" by varying environmental parameters - suggests the possibility of tailoring the catalytic mechanisms of sufficiently small CNPs for specific reactions - e.g., stabilizing sufficiently small CNPs with -OH groups for application in water splitting or the water-gas shift reaction. Such mechanisms would represent direct reaction pathways that do not depend on the annihilation and regeneration of $\mathrm{O}$-vacancies as would be required in bulk ceria or ceria samples exhibiting ideal, unreconstructed, stoichiometric bulk-like surface terminations.

\section{Acknowledgments}

The authors Xing Huang and Matthew J. Beck acknowledge high performance computing resources provided by Center for Computational Sciences at University of Kentucky.

\section{Appendix A. Ce/O atomic ratios in various CNP geometries}

For cubic $\{100\}$-terminated CNPs with $N$ Ce atoms along each edge, there are $4 N^{3}$ Ce atoms and $(2 N-1)^{3}$ "bulk" O atoms internal to the CNP. In the absence of additional $\mathrm{O}$ atoms (e.g., for a Ce-terminated CNP) the ratio of Ce to $\mathrm{O}$ atoms, $n_{C e} / n_{O}$, is:

$$
\frac{n_{C e}}{n_{O}}=\frac{4 N^{3}}{(2 N-1)^{3}}
$$


If the CNP surface is fully $\mathrm{O}$-terminated-that is, if every surface $\mathrm{O}$ lattice site is occupied by one $\mathrm{O}$ atom-there are 4 additional $\mathrm{O}$ atoms at the corners, $12(2 N-1)$ at edges and $6(2 N-1)^{2}$ at facets. Thus, $n_{C e} / n_{O}$ becomes

$$
\frac{n_{C e}}{n_{O}}=\frac{4 N^{3}}{(2 N-1)^{3}+6(2 N-1)^{2}+12(2 N-1)+4}
$$

Figure A.7(a) plots $n_{C e} / n_{O}$ as a function of CNP size for cubic $\{100\}-$ terminated CNPs with either $\mathrm{Ce}-$ or $\mathrm{O}$-terminations. In both cases, $\mathrm{CeO}_{2}$ stoichiometry (that is $n_{C e} / n_{O} \equiv 0.5$ ) is a limiting case, and cannot be achieved for cubic $\{100\}$-terminated CNPs of finite size. Constructing a finite, cubic $\{100\}$-terminated $\mathrm{CNP}$ with exact $\mathrm{CeO}_{2}$ stoichiometry therefore requires either deleting some fraction of $\mathrm{O}$ atoms present in $\mathrm{O}$-terminated CNPs, or deleting Ce atoms from Ce-terminated CNPs.

For octahedral $\{111\}$-terminated with $N$ Ce atoms along each edge, there are $N\left(2 N^{2}+1\right) / 3$ Ce atoms and $4 N(N-1)(N+1) / 3$ "bulk" O atoms. In this case "bulk" $\mathrm{O}$ atoms include those $\mathrm{O}$ atoms present as part of stoichiometric $\{111\}$ surfaces. Note that though $\{111\}$ CNP facets are stoichiometric, their edges and corners are not. For octahedral $\{111\}$-terminated CNPs with no additional $\mathrm{O}$ atoms at edges and corners (this is the Ceterminated case), the ratio of Ce to $\mathrm{O}$ atoms, $n_{C e} / n_{O}$, is:

$$
\frac{n_{C e}}{n_{O}}=\frac{2 N^{2}+1}{4\left(N^{2}-1\right)}
$$

As above if every surface $\mathrm{O}$ lattice site is occupied by one $\mathrm{O}$ atom (the $\mathrm{O}$-terminated limit) there are 24 additional $\mathrm{O}$ atoms at the CNP corners, $24(N-2)$ at edges and $4(N-2)(N-3)$ at facets. In this case the total number of $\mathrm{O}$ atoms becomes

$\frac{4 N(N-1)(N+1)}{3}+24(N-2)+24+4(N-2)(N-3)=\frac{4 N(N+1)(N+2)}{3}$ 
Thus, for octahedral $\{111\}$-terminated CNPs with all surface O lattice sites occupied by $\mathrm{O}$ atoms (this is the $\mathrm{O}$-terminated case), $n_{C e} / n_{O}$ becomes

$$
\frac{n_{C e}}{n_{O}}=\frac{2 N^{2}+1}{4(N+1)(N+2)}
$$

Figure A.7(b) plots $n_{C e} / n_{O}$ as a function of CNP size for octahedral $\{111\}-$ terminated CNPs with either $\mathrm{Ce}^{-}$or O-terminations. As for the $\{100\}-$ terminated CNPs discussed above, exactly stoichiometric finite-sized $\{111\}-$ terminated CNPs can only be constructed by removing $\mathrm{O}$ atoms or Ce atoms from O-terminated or Ce-terminated CNPs, respectively. 

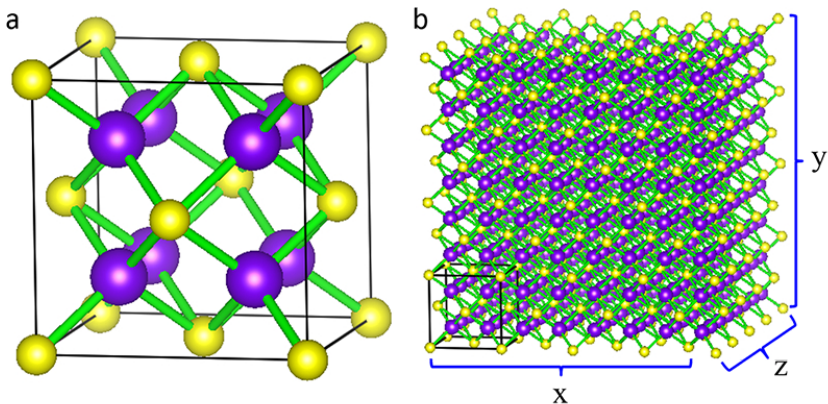

f

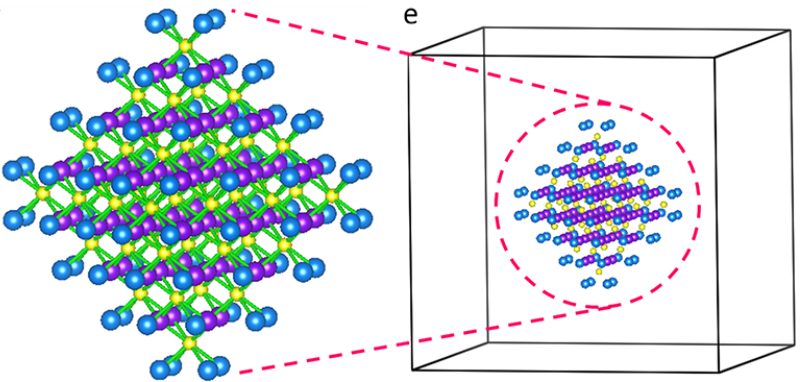

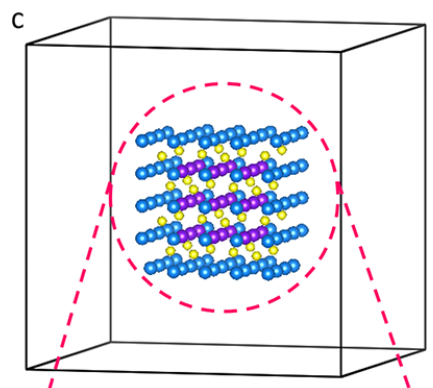

d

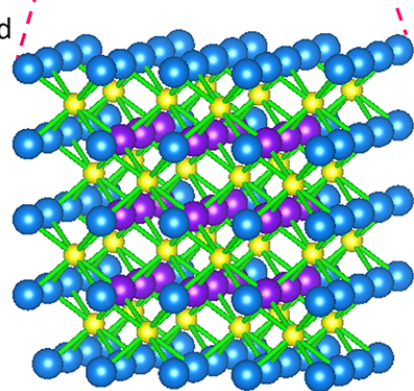

Figure 2: (a) Cubic unit cell of bulk ceria showing cubic fluorite structure. Purple balls are bulk $\mathrm{O}$ atoms, yellow balls Ce atoms, and sticks highlight nearest neighbor coordinations. (b) Schematic illustration of the first step in constructing various CNP structures: the ceria bulk unit cell (highlighted with a black outline) is repeated in space to construct an arbitrarily-sized Ce-terminated CNP. This serves as the basic bulk unit from which to further construct initial CNP structures with different sizes, shapes, and surface configurations. (c) and (d) An example initial structure constructed for $\sim 1 \mathrm{~nm}$ \{100\}-terminated cubic CNPs. The supercell (c) is chosen to create vacuum separation between nanoparticles when periodic boundary conditions are applied. This particular initial CNP structure contains 32 Ce atoms shown as purple balls, 27 "bulk" O atoms shown as yellow balls, and 94 "surface" O atoms occupying every O lattice site at CNP facets, edged, and corners. (e) and (f) An example initial structure constructed for $\sim 1 \mathrm{~nm}\{111\}$-terminated octahedral CNPs. This example initial CNP structure contains 44 Ce atoms shown as purple balls, 80 "bulk" O atoms shown as yellow balls, and 80 "surface" O atoms occupying every $\mathrm{O}$ lattice site at facets, edged, and corners to fully coordinate all Ce atoms. 

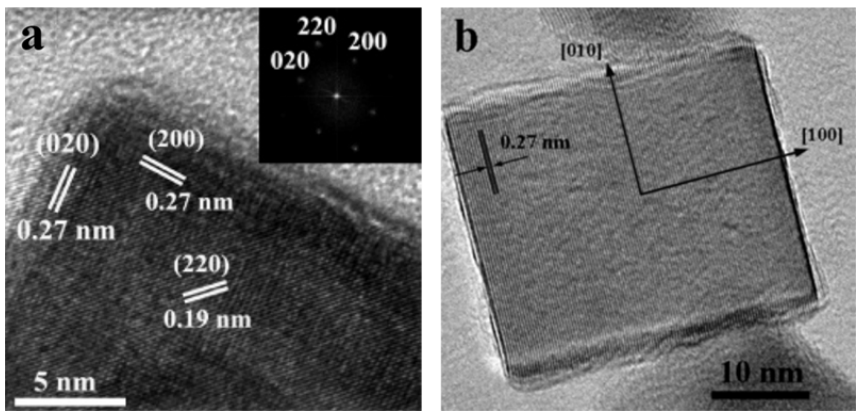

Figure 3: (a) High resolution TEM image of $\{100\}$-terminated cubic CNP highlighting (200) interplanar spacing of $0.27 \mathrm{~nm}$, along with fast Fourier transform (FFT) analysis (inset) showing single crystallinity [? ]. Reprinted with permission from reference [? ]. (b) High resolution TEM image showing \{100\}-terminated cubic CNP and consistent (100) lattice fringes spaced at $0.27 \mathrm{~nm}[?]$. Reprinted with permission from reference [? ]. 


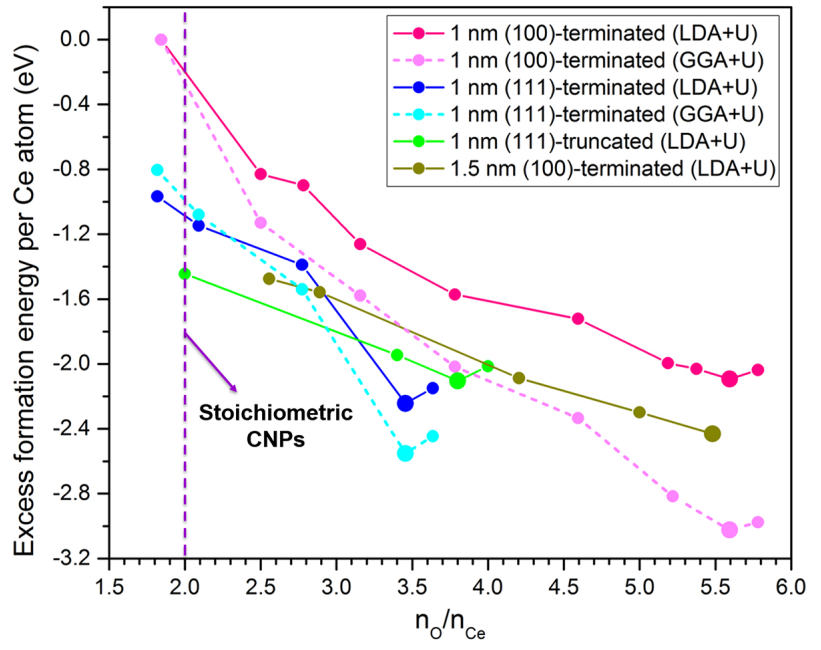

Figure 4: Calculated excess energy of formation per Ce atom $\left(\Delta e_{f}^{C N P}\right.$, see text for details) for CNPs with various shapes, sizes and surface $\mathrm{O}$ concentrations as referenced to the reduced $\mathrm{CNP}, \mathrm{Ce}_{32} \mathrm{O}_{57}$ and at the corrected chemical potential of $\mathrm{O}, \mu_{O}^{*}$. LDA $+U$ results for $\mathrm{U}=5 \mathrm{eV}$ are connected by solid lines, while GGA $+U$ results for $\mathrm{U}=3$ $\mathrm{eV}$ are connected with dashed lines. The most stable CNP structure for each series of calculations is shown with a larger dot. These results demonstrate that sufficiently small CNPs are strongly stabilized by the adsorption of stoichiometrically excess $\mathrm{O}$ atoms, even in the O-lean limit. 


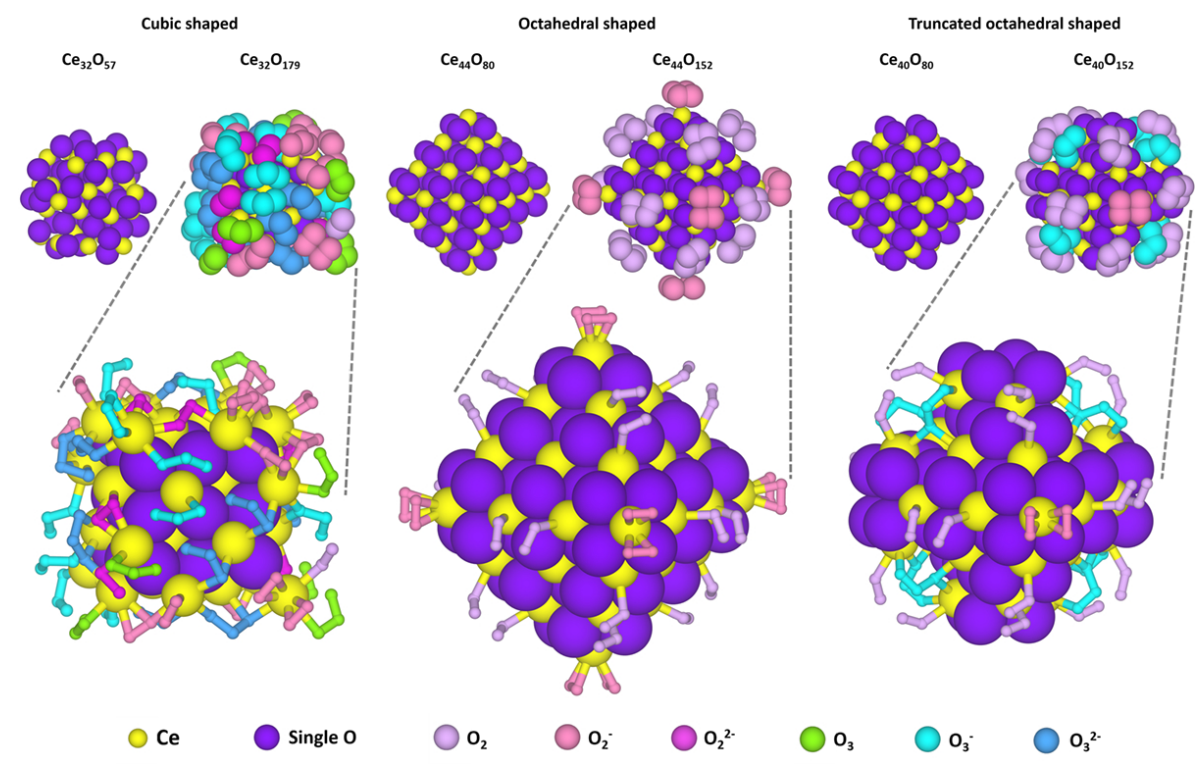

Figure 5: Atomic structures of CNPs with different shapes and compositions as calculated with $\mathrm{LDA}+\mathrm{U}$ for $U_{\mathrm{eff}}=5 \mathrm{eV}$. Small yellow spheres are Ce atoms, purple spheres are individual lattice $\mathrm{O}$ atoms, and blue/pink/green spheres are variously charged $\mathrm{O}$ pairs and triples. The three lower panels are identical to the upper right panels for each shape, but exposing the binding configuration of surface $\mathrm{O}_{x}^{q}$ groups. See text for details. 


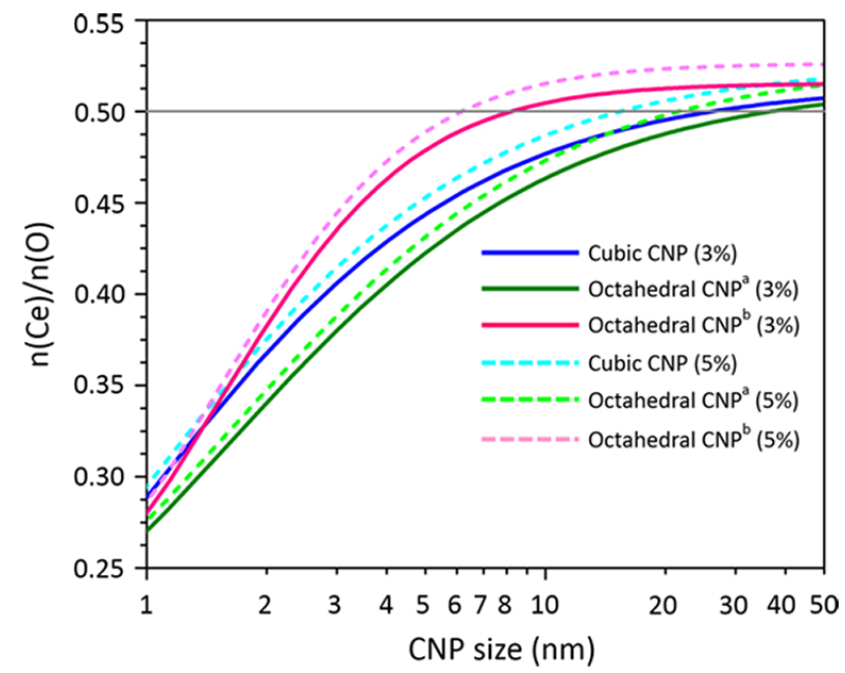

Figure 6: Ratio of Ce to $\mathrm{O}$ atoms in cubic and octahedral CNPs as a function of CNP size. Cubic CNP and octahedral $\mathrm{CNP}^{a}$ structures are fully oxidized, while octahedral $\mathrm{CNP}^{b}$ structures exhibit stoichiometric $\{111\}$ facets, but fully coordinated (oxidized) edges and corners. 

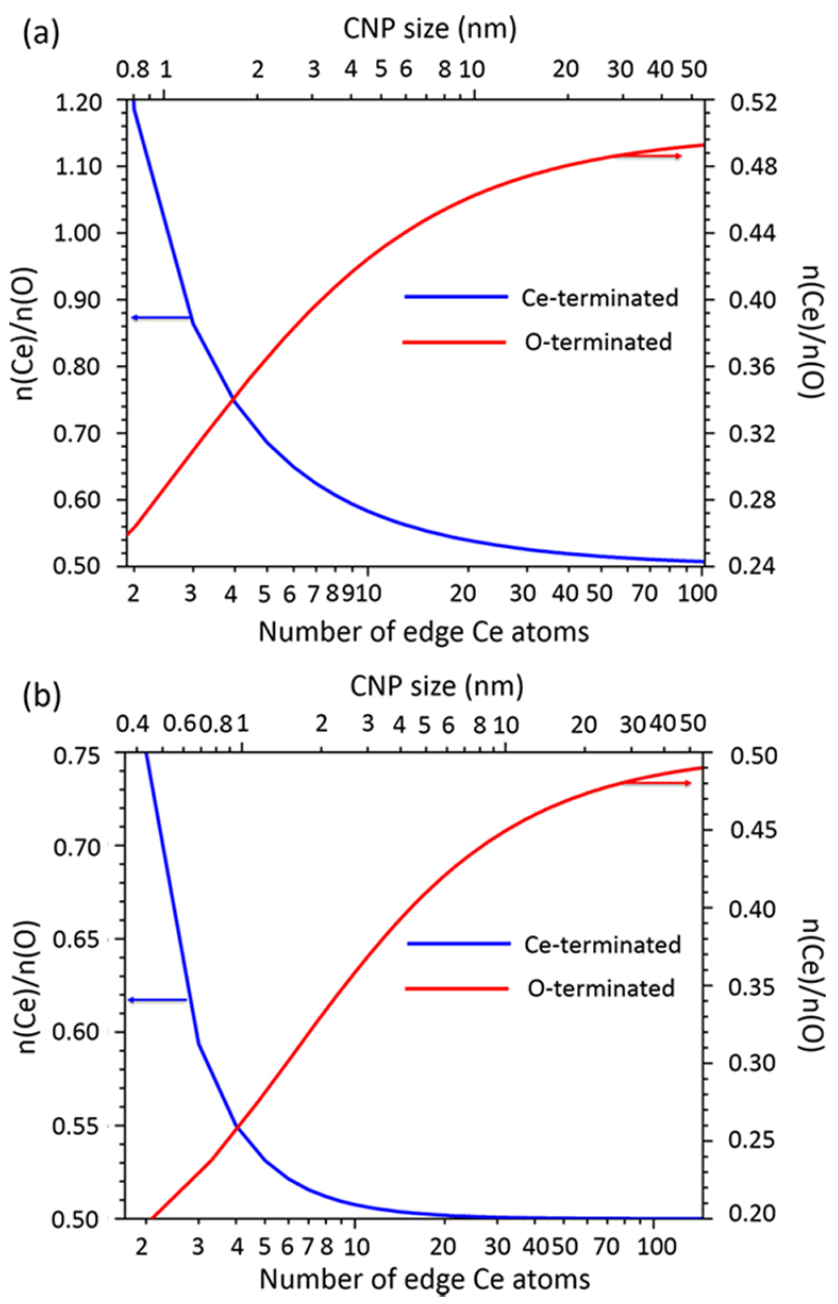

Figure A.7: (a) $n(\mathrm{Ce}) / n(\mathrm{O})$ as a function of CNP size for Ce-terminated (blue) cubic CNPs and O-terminated (red) cubic CNPs. (b) $n(\mathrm{Ce}) / n(\mathrm{O})$ as a function of CNP size for Ce-terminated (blue) octahedral CNPs and O-terminated (red) octahedral CNPs. 